\title{
Recovering Chemistry at Atomic Resolution using Multi-Modal Spectroscopy
}

Jonathan Schwartz ${ }^{1}$, Yi Jiang ${ }^{2}$, Zichao Wendy $\mathrm{Di}^{3}$, Tao Ma ${ }^{4}$, Huihuo Zheng ${ }^{5}$, Steve Rozeveld ${ }^{6}$ and Robert Hovden $^{1}$

${ }^{1}$ Department of Materials Science and Engineering, University of Michigan, United States, ${ }^{2}$ Advanced Photon Source, Argonne National Laboratory, Lemont, IL 60439, USA, United States, ${ }^{3}$ Mathematics and Computer Science Division, Argonne National Laboratory, United States, ${ }^{4}$ Michigan Center for Materials Characterization, United States, ${ }^{5}$ Argonne Leadership Computing Facility, Argonne National Laboratory, United States, ${ }^{6}$ Dow Chemical Co., United States

Advances in scanning transmission electron microscopy has enabled the simultaneous collection of multiple imaging modalities including elastic and inelastic scattering processes. The chemical composition of materials can be probed down to atomic resolution via energy dispersive x-ray (EDX) and electron energy loss spectroscopy (EELS). However high-radiation doses are required (e.g. $10^{6}$ e/ $/ \AA^{2}$ ) which limits signal-to-noise (SNR) and prevents spectroscopy of beam sensitive specimens. High-angle annular dark-field (HAADF) micrographs contain contrast proportional to the atomic number $(\mathrm{Z})$ via Rutherford scattering [1] producing images with high SNR at lower doses (e.g. $10^{3}$ e/ $\AA^{2}$ ). Unfortunately, Z-contrast imaging can only distinguish well separated atoms with noticeably different atomic numbers. Conventionally each modality-HAADF and EDX/EELS - is analyzed separately, however these imaging modes provide useful complementary information about the specimen [2].

Here we introduce multi-modal spectroscopy, a technique that offers high SNR recovery of material chemistry by leveraging correlated information encoded within both HAADF and EDX / EELS. We demonstrate chemical recovery at the nanoscale (Fig. 1) in commercial CoS catalysts for oxygen-reduction [3]. Poisson noise in the raw EDX maps (Fig. 1a-c) is virtually eliminated (Fig. 1e-g) while maintaining the unique oxide and core phases at the interface. Multi-modal spectroscopy also succeeds at atomic resolution as shown on a ferromagnetic Strontium Titanate and Lithium Manganese Oxide (STO/LMO) interface (Fig. 2). We can recover light elements such as Oxygen $(\mathrm{O})$ (Fig. 2d) that are weakly present in the simultaneous HAADF (Fig. 2a). These results demonstrate multi-modal spectroscopy can substantially improve the quality of under-measured spectral maps and ensure accurate chemical recovery even under minimal dose. In both cases, we see that multi-modal spectroscopy significantly improves the chemical maps by boosting SNR.

We recover chemical maps by solving an optimization problem that seeks a solution that strongly correlates with (1) the HAADF modality containing high SNR, (2) the chemically sensitive spectroscopic modality, and (3) is maximally sparse in the gradient domain. These three terms define our multi-modal approach to surpassing traditional dose limits. First, we assume a forward model where the simultaneous HAADF is the linear combination of elemental distributions $\left(\boldsymbol{x}^{\gamma}\right.$ where $\left.\gamma \in[1.4,2]\right)$. We ensure the recovered signals maintains a high-degree of data fidelity with the initial measurements by using maximum log-likelihood for EDX measurements dominated by low-count Poisson statistics. Finally, we utilize channel-wise total variation (TV) regularization to enforce a sparse gradient magnitude, which reduces noise and promotes image smoothness - an approach popularized by the field of Compressed Sensing (CS) [5]. The overall optimization function results as following: 


$$
\Psi(\boldsymbol{x})=\arg \min _{\boldsymbol{x}} \frac{\lambda_{1}}{2}\left\|\boldsymbol{b}_{H}-\sum_{i} \boldsymbol{x}_{i}^{\gamma}\right\|_{2}^{2}+\lambda_{2} \sum_{i}\left(\boldsymbol{x}_{i}-\boldsymbol{b}_{i} \log \left(\boldsymbol{x}_{i}\right)\right)+\lambda_{3} \sum_{i}\left\|\boldsymbol{x}_{i}\right\|_{T V}
$$

Where $\boldsymbol{b}_{H}$ is the HAADF image, $\lambda_{i}$ are regularization parameters, $\boldsymbol{b}_{\mathrm{i}}$ and $\mathbf{x}_{i}$ are the measured and recovered chemical maps.

As a result, multi-modal spectroscopy reveals chemistry and enhances contrast at significantly lower electron doses than traditional approaches_-demonstrated for nano- (Fig. 1) and atomic-scale (Fig. 2) mapping.

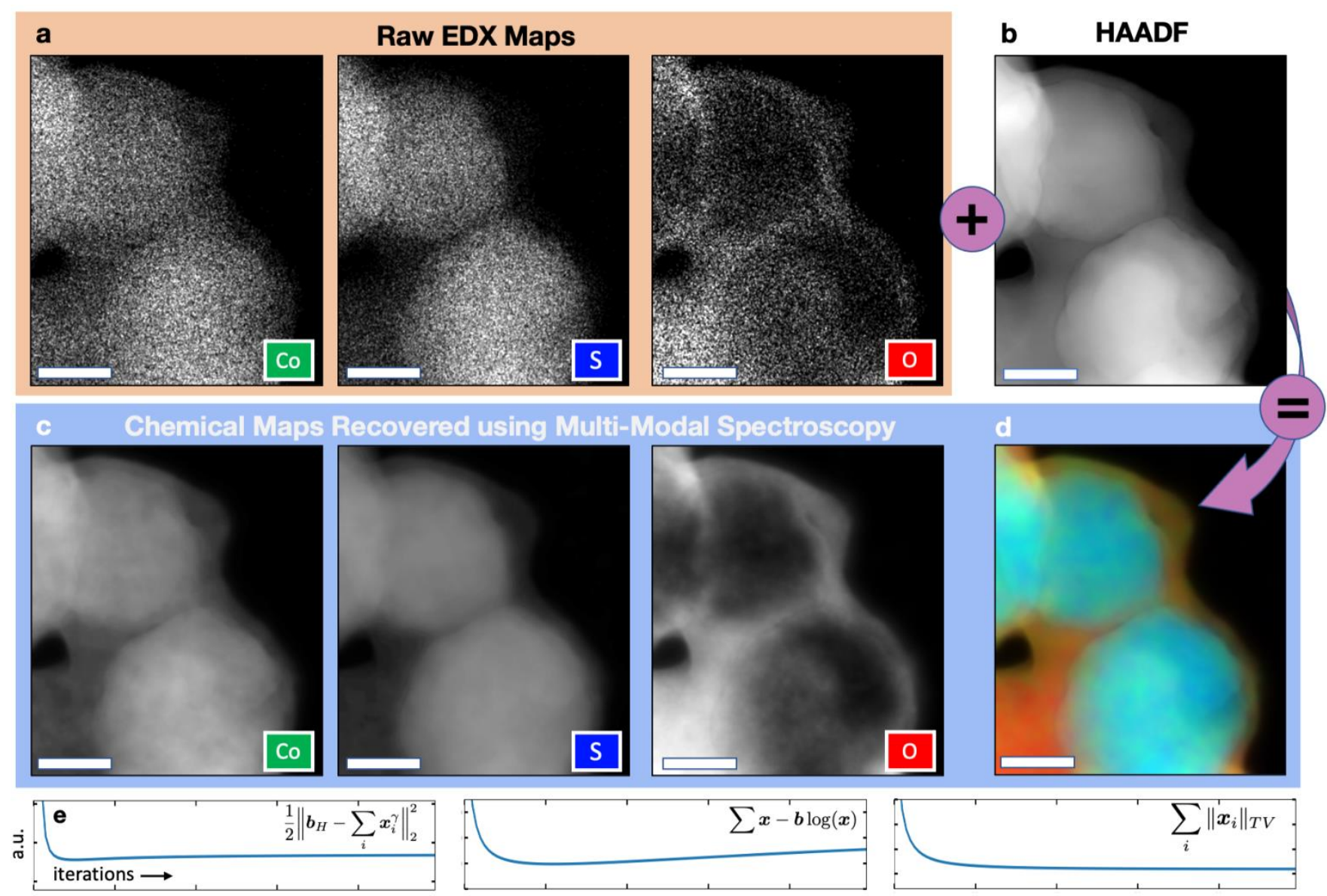

Figure 1. Nanoscale multi-modal EDX chemical recovery of CoS catalysts. a, The raw EDX chemical maps for the Co, S, and $\mathrm{O}$ elemental distributions. b, The simultaneous HAADF micrograph of the CoS nanoparticle. c, The multi-modal reconstructions for the elemental distributions. d, EDX overlay of the $\mathrm{Co}, \mathrm{S}$, and $\mathrm{O}$ maps. Scale bar, $30 \mathrm{~nm}$. e, Convergence of each component within the cost function as the recovery process proceeds. 

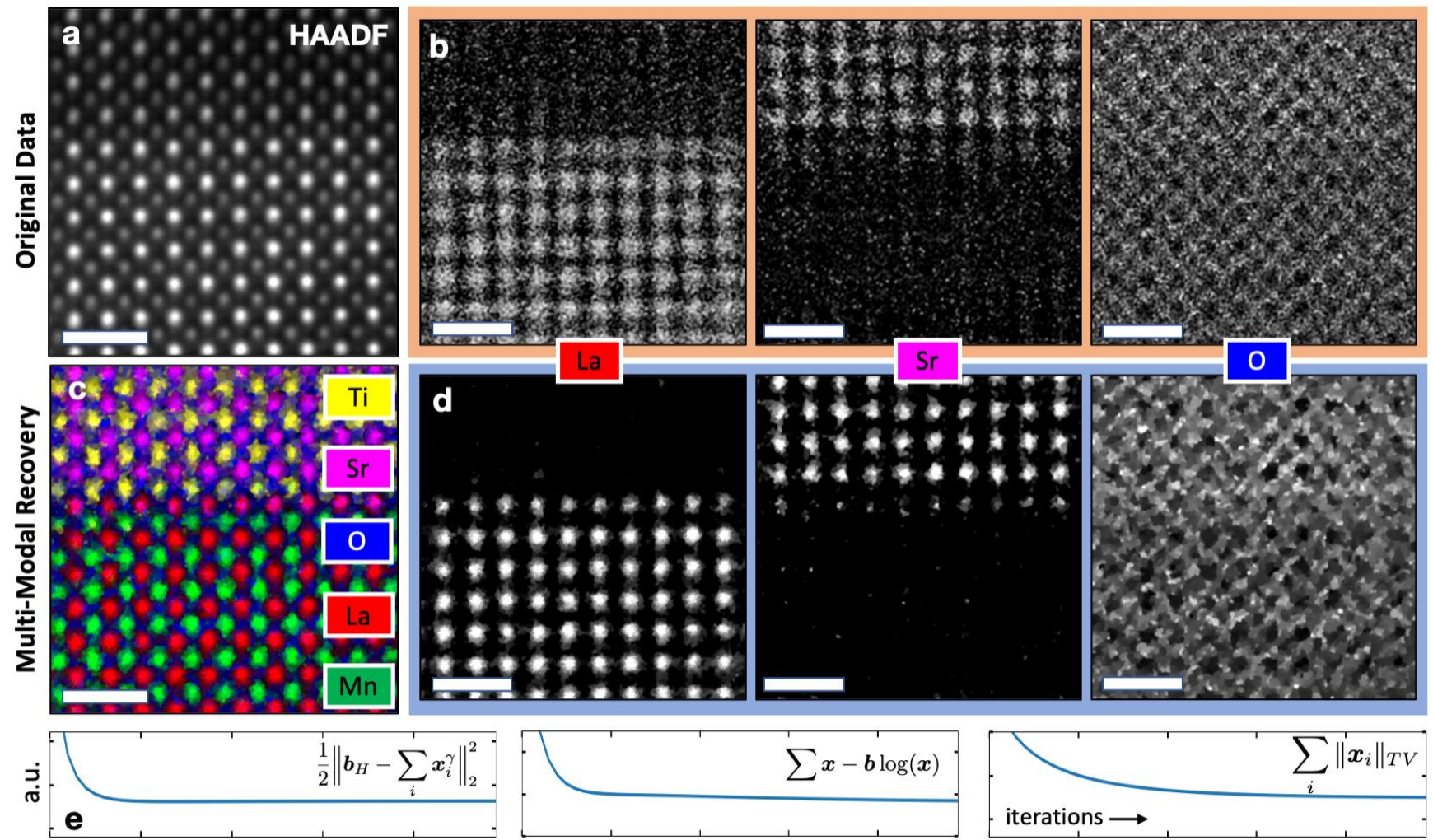

Figure 2. Atomic-resolution EDX elemental mapping of a STO/LMO interface. a, Simultaneous HAADF map averaged over 100 acquisition frames [6]. b, The original EDX maps for La, O, and Sr. c, The EDX overlay of Ti, Sr, O, La, and Mn. d, Multi-modal reconstructions of La, O, and Sr. Scale bar, $1 \mathrm{~nm}$. e, Convergence of each component within the cost function as the recovery process proceeds.

\section{References}

[1] O. Krivanek, et. al. Nature 464, 571 (2010).

[2] T. Thersleff, S. Budnyk, L. Drangai, A. Slabon, Ultramicroscopy 219, 113116 (2020).

[3] S. Rozeveld, W. Lee, P. Longo, Microscopy and Microanalysis 26 (S2) 1248 (2020).

[4] Y. Jiang, et. al. Nature 559, 343 (2018).

[5] D. Donoho IEEE Trans. Inf. Theor. 52, 1289 (2006).

[6] B. Savitzky, et. al. Ultramicroscopy 191, 56 (2018). 